\title{
Intraductal Radiofrequency Ablation (RFA) for Pancreatic Cancer: Getting in Under the Wire?
}

\author{
Brian C. Brauer ${ }^{1}$
}

Published online: 25 July 2015

(C) Springer Science+Business Media New York 2015

Since pancreatic carcinoma, an aggressive cancer often unresectable at initial diagnosis, is curable by complete surgical removal in only 10-20\% of cases [1], palliative therapy, primarily chemotherapy, is therefore the mainstay of treatment. Newer regimens combining chemotherapy with either external beam or stereotactic body radiation therapy (SBRT) and more intense regimens such as 5-fluorouracil, irinotecan, and oxaliplatin (FOLFIRINOX) are ongoing. Nevertheless, even with the best care, survival is dismal, especially in those with unresectable disease [2,3].

Although biliary obstruction, commonly complicating disease involving the pancreatic head, can be stented successfully during endoscopic retrograde cholangiopancreatography (ERCP), stent occlusion is a frequent problem, even with the newer metallic stents, whether covered or uncovered. Stent occlusion can be complicated by jaundice, cholangitis, and delay in chemotherapy or other treatments. Since the prognosis for survival with locally advanced or metastatic pancreatic carcinoma is poor, endoscopic therapy of advanced disease is merely palliative.

Radiofrequency ablation (RFA) delivered primarily by the intraoperative and percutaneous routes has been used for years to treat a variety of tumors. The development of new "over-the-wire" endobiliary RFA catheters compatible with existing electrosurgical units eases the application of RFA while reducing its cost [4]. The endobiliary RFA device was originally designed to be applied to malignant

Brian C. Brauer

Brian.brauer@ucdenver.edu

1 Division of Gastroenterology and Hepatology, University of Colorado Anschutz Medical Campus, 1635 Aurora Ct, Mailstop F-735, Room AIP 2.031, Aurora, CO 80045, USA biliary strictures to slow intraductal growth and improve stent patency. In this issue of Digestive Diseases and Sciences, Kallis et al. [5] sought to determine whether endoscopically delivered RFA prolongs stent patency and survival in patients with pancreatic carcinoma.

In a large-volume center routinely performing RFA for pancreatic carcinoma, the authors compared, using a retrospective case-control analysis, 23 patients with biliary obstruction due to unresectable pancreatic cancer undergoing endoscopic RFA/SEMS with 46 controls undergoing SEMS alone. The authors' uniform approach to all study subjects, including use of the same stent type and the same chemotherapy regimens, provides a reasonable basis for comparison. The primary endpoint was patient survival with secondary endpoints stent patency and procedure-related safety and tolerability. A significant survival benefit was observed in patients treated with RFA and SEMS (226 vs 123.5 days, $p=0.01$ ), although no difference in stent patency was observed (median 472 vs 324 days, $p=0.669$ ). RFA was independently predictive of survival at 90 and 180 days. In both groups, patients died primarily from disease progression and carcinomatosis, but not from the direct effects of stent occlusion.

What is the mechanism by which RFA prolongs survival? Although it has been postulated that RFA decreases intimal hyperplasia [6], this does not explain increased survival in the absence of increased stent patency. Hänsler et al. [7] reported in a study of 10 patients with primary or secondary liver tumors undergoing RFA increased cytologic tumorspecific $\mathrm{T}$ cell stimulation suggestive of an immunological mechanism for increased survival. den Brok et al. [8] also reported increased immune response to RFA in a mouse model, suggesting that immune activation by RFA may enhance tumor suppression. Although enhanced immunogenicity may very well explain the increased survival, this 
was not specifically examined in the discussed study and thus remains speculative.

Although the controls were carefully matched, including by type of chemotherapy, controls were taken from an earlier time period (years 2005-2010 for controls vs 2009-2011 for treatment group). It is possible that improved survival in the RFA group may be due to differences in management of patients during these time periods including improved or earlier administration of chemotherapy and improved management of complications. Nonetheless, RFA was safe and well tolerated with no major adverse effects.

Larger, prospective randomized trials will be vital not only to corroborate the findings of Kallis et al. but also to elucidate the mechanism involved. It would also be helpful to determine prospectively whether or not stent patency is increased in those undergoing RFA. If survival is improved independently of stent patency, further investigation of the immunological effects of RFA should be undertaken. It is not known whether the dose of RFA via the endobiliary route is sufficient to create an immunological response as observed in other studies. It might also be useful to measure tumor burden, either by cross-sectional imaging or by functional positron emission tomography, to determine whether improved survival (assuming the result is reproducible) is associated with decreased tumor burden. Even in the absence of increased survival, it is important to identify the effect of RFA on tumor burden.

It would also be useful, and perhaps more important, to examine the effect of RFA on patients with perihilar cholangiocarcinoma, a disease not studied by the authors which behaves somewhat differently than does ductal adenocarcinoma. Overall survival is longer [9], with much of the morbidity and mortality related to stent occlusion, undrained segments, and recurrent bouts of cholangitis. Maintaining good drainage in these patients is a key to survival. A technique that slows intraductal growth and helps to prolong drainage such as endobiliary RFA would thus be welcomed in this population. A recent retrospective study that included a small number of patients with cholangiocarcinoma suggested a survival benefit [10].

From a practical standpoint, RFA is well tolerated and easy to use. Given that catheters are compatible with most currently used electrosurgical generators, the investment is small; hence, most practitioners performing ERCP can easily incorporate the technology into their practice. The current practice at our institution is to use RFA in patients who have early stent occlusion and in select malignant hilar stricture cases prone to early stent occlusion or recurrent cholangitis. Given the safety of the device and its ease of use, combined with the efficacy demonstrated in this small study, we advocate gastrointestinal practitioners who have the technology at their disposal to rapidly adopt the procedure.

\section{References}

1. Bilimoria KY, Bentrem DJ, Ko CY, et al. Validation of the 6th edition AJCC pancreatic cancer staging system: report from the national cancer database. Cancer. 2007;110:738-744.

2. Chan SL, Chan ST, Chan EH, He Z-X. Systemic treatment for inoperable pancreatic adenocarcinoma: review and update. Chin J Cancer. 2014;33:267-276.

3. Tozzi A, Comito T, Alongi F, et al. SBRT in unresectable advanced pancreatic cancer: preliminary results of a mono-institutional experience. Radiat Oncol. 2013;8:148. (London, England).

4. Figueroa-Barojas P, Bakhru MR, Habib NA, et al. Safety and efficacy of radiofrequency ablation in the management of unresectable bile duct and pancreatic cancer: a novel palliation technique. J Oncol. 2013;2013:910897.

5. Kallis Y, Phillips N, Steel A, et al. Analysis of endoscopic radiofrequency ablation of biliary malignant strictures in pancreatic cancer suggests potential survival benefit. Dig Dis Sci. (Epub ahead of print). doi:10.1007/s10620-015-3731-8.

6. Steel AW, Postgate AJ, Khorsandi S, et al. Endoscopically applied radiofrequency ablation appears to be safe in the treatment of malignant biliary obstruction. Gastrointest Endosc. 2011;73:149-153.

7. Hänsler J, Wissniowski TT, Schuppan D, et al. Activation and dramatically increased cytolytic activity of tumor specific $T$ lymphocytes after radio-frequency ablation in patients with hepatocellular carcinoma and colorectal liver metastases. World $J$ Gastroenterol. 2006;12:3716-3721.

8. den Brok MH, Sutmuller RP, van der Voort R, et al. In situ tumor ablation creates an antigen source for the generation of antitumor immunity. Cancer Res. 2004;64:4024-4029.

9. Koerkamp BG, Fong Y. Outcomes in biliary malignancy. J Surg Oncol. 2014;110:585-591.

10. Sharaiha RZ, Natov N, Glockenberg KS, Widmer J, Gaidhane M, Kahaleh M. Comparison of metal stenting with radiofrequency ablation versus stenting alone for treating malignant biliary strictures: Is there an added benefit? Dig Dis Sci. 2014;59: 3099-3102. 